\title{
Influence of Random, Pile-up and Scatter Corrections in the Quantification Properties of Small-Animal PET Scanners
}

\author{
E. Vicente ${ }^{1}$, M. Soto-Montenegro ${ }^{2}$, S. España ${ }^{1}$, J. L. Herraiz ${ }^{1}$, E. Herranz ${ }^{1}$, J. J. Vaquero ${ }^{2}$, M. \\ Desco $^{2}$, J. M. Udías ${ }^{1}$ \\ ${ }^{1}$ Grupo de Física Nuclear, Dpto. Física Atómica, Molecular y Nuclear, Universidad \\ Complutense de Madrid, Spain \\ ${ }^{2}$ Unidad de Medicina y Cirugía Experimental, Hospital GU “Gregorio Marañón”, Madrid, Spain
}

\begin{abstract}
The potential of PET imaging for pre-clinical studies will be fully realized only if repeatable, reliable and accurate quantitative analysis can be performed. The characteristic blurring of PET images due to positron range and non co-linearity, as well as random, pile-up and scatter contributions, that may be significant for fully 3D PET acquisitions of small animal, make it difficult their quantitative analysis. In this work specific activity versus specific counts in the image calibration curves for 3D-OSEM reconstructions from a commercially available small animal PET scanner are determined. Both linear and non-linear calibration curves are compared and the effect of corrections for random and scatter contributions are studied. To assess the improvement in the calibration procedure when scatter and random corrections are considered, actual data from a rat tumor pre- and postcancer therapy are analyzed. The results show that correcting for random and scatter corrections can increase the sensitivity of PET images to changes in the biological response of tumors by more than $15 \%$, compared to uncorrected reconstructions.
\end{abstract}

\section{INTRODUCTION}

There is growing evidence [1]-[4] that ${ }^{18} \mathrm{~F}$-fluoro-2deoxyglucose (FDG) PET studies could have significant impact on the management of cancer patients. The evaluation of therapy response is usually done by computed tomography $(\mathrm{CT})$, ultrasonography or magnetic resonance imaging (MRI). These imaging procedures allow the detection of changes in tumor size or volume, but do not provide tumor-specific functional information. The identification of (non-)responders to toxic treatment with these conventional techniques is far from optimal [5]-[8]. For example, it is difficult to distinguish residual disease from necrosis.

PET imaging is able of disentangle differences in the metabolism of tissues. Thus, PET imaging with FDG is rapidly becoming a major tool in oncology because cancer cells have a much higher rate of glycolysis than

Manuscript received November 15, 2007.

E.Vicente email: evicente@nuclear.fis.ucm.es,

M. Soto-Montenegro email: marisa@mce.hggm.es,

S. España e-mail: samuel@nuclear.fis.ucm.es,

J. L. Herraiz e-mail: joaquin@nuclear.fis.ucm.es.

E. Herrnaz e-mail: eherranz@nuclear.fis.ucm.es,

J.J.Vaquero email: juanjo@mce.hggm.es,

M. Desco e-mail: desco@mce.hggm.es;

J.M. Udias e-mail: jose@nuc2.fis.ucm.es non-neoplastic cells and therefore they have increased uptake of glucose and high signal on FDG-PET imaging. As functional changes are likely to precede anatomical ones, FDG PET could meet the need for timely and accurate non-invasive techniques that monitor response to therapy.

The impact of FDG-PET imaging in cancer therapy effectiveness evaluation for preclinical studies depends on the availability of accurate data quantification. Indeed, response of lesions or tumors would only be assessed if the quantification of the reconstructed activity were stable with time and repeatable for different conditions of activity and rates and, correspondingly, different ratios of random and scatter counts.

Typically, PET scanners exhibit a non flat activity versus count response, with two components: a linear one for which the count rate is linearly proportional to the activity in the field of view, as is the case of counts coming from 'true' and scatter coincidences and a nonlinear contribution, coming for instance from dead time and pile-up effects as well as random count contributions [9]. Assessing the importance of non-linear terms is essential for the calibration of the scanner. In this work, in order to estimate the contribution of non linear terms, linear and nonlinear specific activity versus specific counts in the image calibration curves for 3D-OSEM reconstructions of a commercial small animal PET scanner were obtained and Standard Uptake Values (SUV) were computed for two different reconstruction methods, one taking into account corrections for random, pile-up and scatter contributions and another without these corrections. The comparison of the results of these two methods would allow to disentangle whether these corrections in the reconstruction and calibration methods have any noticeable effect in the ability of PET images for determining changes in tumor activity.

\section{MATERIALS \& MethodS}

\section{A. Scanner description}

Data from the high-resolution small animal rPET [10] scanner (SUINSA Medical Systems) were employed. This PET system has four detectors arranged in two orthogonal pairs which rotate $180^{\circ}$. Each detector comprises a single layer array of $30 \times 30$ MLS crystals $\left(1.5 \times 1.5 \times 12 \mathrm{~mm}^{3}\right)$ optically coupled to a Hamamatsu H8500 flat-panel PS-PMT. 
The scanner has a ring diameter of $160 \mathrm{~mm}$ with effective transverse and axial field of view of $44.8 \mathrm{~mm}$. The central point sensitivity at the center of the field of view (cFOV) is $2.1 \%(762.2 \mathrm{cps} / \mu \mathrm{Ci})$, the volumetric spatial resolution (in cFOV) is $3.4 \mathrm{~mm}^{3}$ and the average energy resolution is $17 \%$. [11].

\section{B. Calibration acquisitions and analysis}

Rat (5 cm diameter, $15 \mathrm{~cm}$ in length) and mouse (2.5 $\mathrm{cm}$ diameter, $7 \mathrm{~cm}$ in length) size cylinders were filled with FDG. The initial activity was known from an external independent calibration with a well-counter with accuracy of \pm 5 . A total of 125 consecutive acquisitions of 5 minutes were taken with both cylinders. This allowed spanning a large range of both specific activities per unit volume as well as global activity registered by the scanner. Both cylinder acquisitions were reconstructed with a 3D-OSEM procedure [11] with different assumptions. In one of them, there is no attempt to correct for random counts, scatter or pile-up in the acquisition data. In the other one (improved 3D-OSEM), corrections for randoms, scatter and pile-up are incorporated in the reconstruction algorithm [13].

After reconstruction, several regions of interest (ROIs) of different sizes were chosen in the interior of both cylinders and the specific counts per cubic centimeter and second ( $\mathrm{cps} / \mathrm{cc})$ obtained from the reconstruction were compared against the known specific activity $(\mathrm{uCi} / \mathrm{cc})$ in the inner region of both cylinders. The data obtained from both cylinders was fit by

i) a linear calibration curve, looking for the proportionality between the specific rate measured in the image and the specific activity known for each acquisition. A linear fit implicitly assumes that non linear effects (like random and pile-up contributions) can be neglected.

ii) a non linear fit that assumes that the specific measured rate can depend both on the specific activity and its square, for instance due to contribution of random counts.

\section{Rat acquisitions and analysis}

$300 \mathrm{~g}$ rats with a subcutaneous tumor on the inguinal area of around $150 \mathrm{~mm}^{3}$ in size (see Fig. 1 and Fig. 2) were injected with $2 \mathrm{mCi}$ of FDG and scanned for 15 minutes per bed (total studies have 2 or 3 beds) after one hour uptake. To prevent partial volume effects, a small region (from 0.013 to $0.15 \mathrm{~mm}^{3}$ ) well inside the tumor was considered and the average specific activity (in $\mathrm{uCi} / \mathrm{cc}$ ) for this region was determined both prior to radiotherapy treatment and just a few days after therapy. In order to investigate whether dealing with corrections in the reconstruction and calibration methods has measurable effects in the PET capability of determining changes in tumor activity, standard uptake values (SUVs) [14]-[17] were computed as a measure of the biologically enhanced fixation of the radiotracer. To obtain dimensionless SUV values, the activity measured in the ROI is divided by the average specific activity determined from the ratio of the injected dose and the volume of the animal given by weight assuming unity density for the rat, that is:

$$
\mathrm{SUV}=\frac{\mathrm{C}}{\mathrm{D} / \mathrm{W}}
$$

being $C(\mathrm{uCi} / \mathrm{cc})$ the activity concentration in the ROI, $D$ $(\mathrm{uCi})$ the injected dose corrected by uptake time and $w(\mathrm{~g})$ the rat weight, using $1 \mathrm{~g} / \mathrm{cc}$ for the rat density.

As it is well known [16],[17] variations in uptake values are often seen, due to differences in FDG uptake or uncertainties in the injected dose. In order to minimize these effects, uptake values were referenced to whole body (wb) uptake values, determined from the specific activity averaged over a ROI that spans the whole body of the rat. By definition, whole body uptake values should be unity, but variations around unity were observed depending on the acquisition and the calibration/reconstruction method employed.

We also present SUV values referenced to one of the testicles since it showed a homogenous FDG distribution that was not influenced by the treatment and was easily visible in all the images.

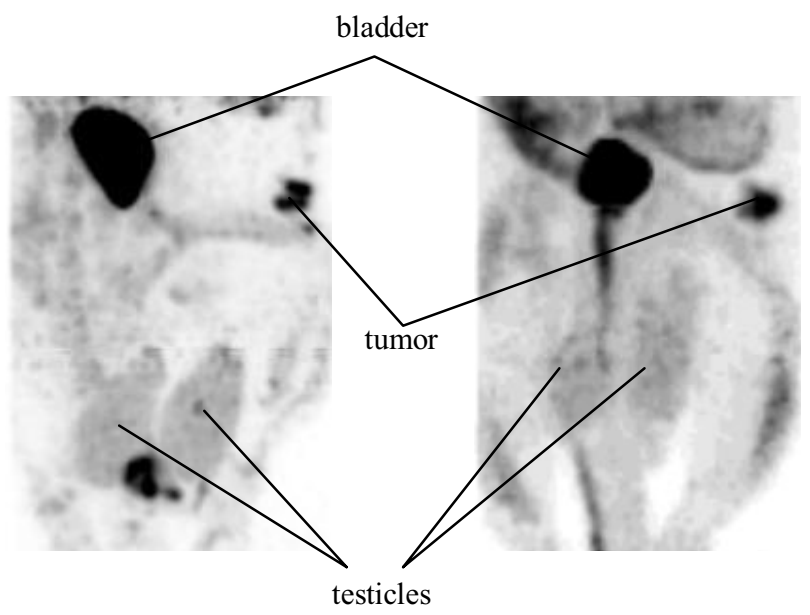

Fig. 1. PET images of a rat with tumor reconstructed with 3D-OSEM. Left: Baseline scan (pre-radiotherapy image of the tumor). Right: Follow-up scan (image of the rat tumor five days after therapy).

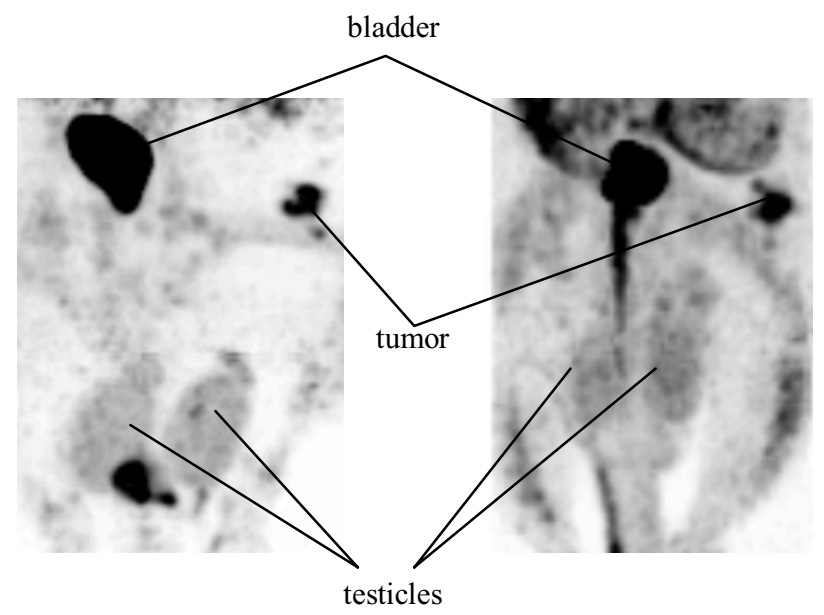

Fig. 2. PET images of the same rat with tumor of Fig. 1 reconstructed with the improved 3D-OSEM code. Left: Baseline scan (preradiotherapy image of the tumor). Right: Follow-up scan (image of the tumor rat five days after therapy). 


\section{RESULTS AND DISCUSSION}

\section{A. Calibration fits}

The difference of the results of the linear fit from the non-linear (quadratic) one was computed for both reconstruction methods. We obtained deviations of $11 \%$ for the standard 3D-OSEM procedure and 5\% for the improved 3D-OSEM. Fig. 3 shows the quadratic fit (nonlinear fit, green line) and the linear one (linear fit 1, yellow line) computed from the whole calibration data range and another linear fit (linear fit 2, red line) calculated from activity concentrations smaller than 63 $\mathrm{uCi} / \mathrm{cc}$, corresponding to the maximum range of tumor data. The parameters of all of fits are quoted in table I. The larger values of the coefficients for the improved 3DOSEM method are due to the fact that a smaller number of counts survive in the image compared to the standard reconstruction, after the (iterative) removal of, mostly, detector scatter counts [13].
TABLE I

PARAMETERS OF CALIBRATION FITS

$$
Y=A_{1} \cdot X+A_{2} \cdot X^{2}\left\{\begin{array}{l}
{[X]=(c p s / c c)} \\
{[Y]=(u C i / c c)}
\end{array}\right.
$$

\begin{tabular}{c||cc|cc}
\multicolumn{1}{c||}{} & \multicolumn{2}{c|}{ 3D-OSEM } & \multicolumn{2}{c}{ Improved 3D-OSEM } \\
Fit & $\begin{array}{c}\mathbf{A}_{\mathbf{1}} \\
(\mathbf{u C i} / \mathbf{c p s})\end{array}$ & $\begin{array}{c}\mathbf{A}_{\mathbf{2}} \\
\left(\mathbf{u C i} \cdot \mathbf{c c} / \mathbf{c p s}^{2}\right)\end{array}$ & $\begin{array}{c}\mathbf{A}_{\mathbf{1}} \\
(\mathbf{u C i} / \mathbf{c p s})\end{array}$ & $\begin{array}{c}\mathbf{A}_{\mathbf{2}} \\
\left(\mathbf{u C i} \cdot \mathbf{c c} / \mathbf{c p s}^{2}\right)\end{array}$ \\
\hline $\begin{array}{c}\text { Non- } \\
\text { linear }\end{array}$ & $1.67 \cdot 10^{-4}$ & $1.48 \cdot 10^{-11}$ & $1.07 \cdot 10^{-3}$ & $3.30 \cdot 10^{-10}$ \\
Linear 1 & $1.50 \cdot 10^{-4}$ & & $1.07 \cdot 10^{-3}$ & \\
Linear 2 & $1.63 \cdot 10^{-4}$ & & $1.04 \cdot 10^{-3}$ &
\end{tabular}

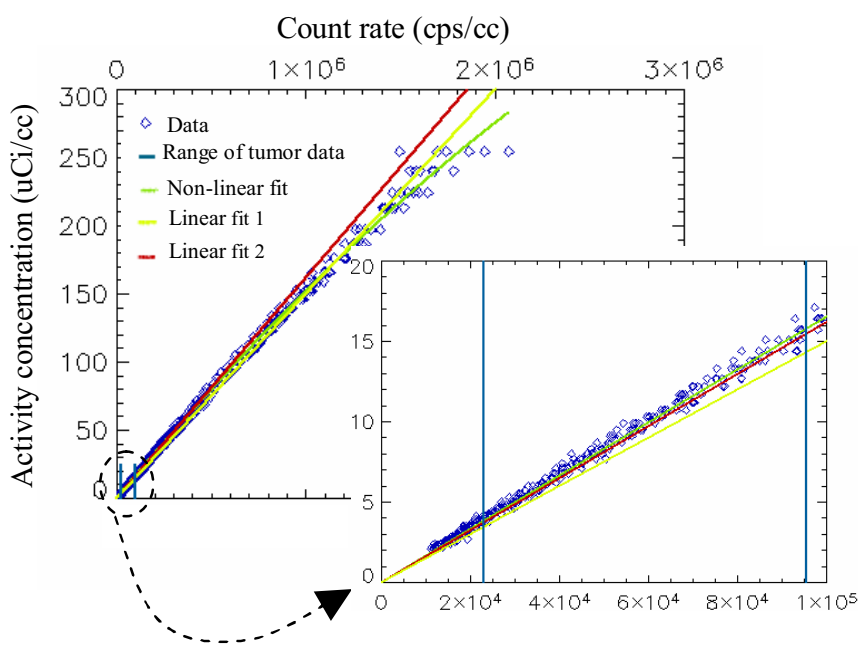

(a)

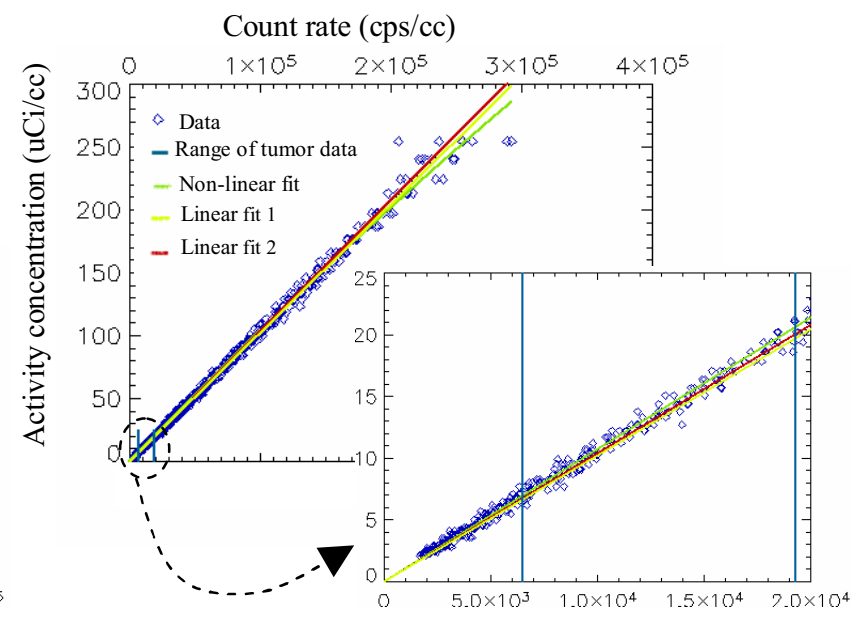

(b)

Fig. 3. Linear and non-linear fits for 3D-OSEM (a) and improved 3D-OSEM (b) reconstruction methods: Counts per cubic centimeter and second $(\mathrm{cps} / \mathrm{cc})$ vs. activity concentration. The actual range of tumor data lies in between the blue vertical thick bars (see zoom of this region in the small insert).

In Fig. 3 (a) (3D-OSEM) we can see that the linear fit 1 does a good job for the higher activity concentrations but not so much for the lower ones. Linear fit 2 seems more suitable for lower concentrations $(<63 \mathrm{uCi} / \mathrm{cc})$, where our tumor data are distributed. Differences between the quadratic fit and the linear fit 2 (for the reduced range of activity concentrations) are about $2 \%$. Fig. 3 (b) (improved 3D-OSEM) shows that there are not significant differences between both linear fits in this case. In what follows we present only SUV values obtained from the linear calibration fit 2 , that uses only data within the range were our tumor data are distributed, activity concentrations below $63 \mathrm{uCi} / \mathrm{cc}$. SUV values were also calculated with the nonlinear (quadratic) calibration but the deviations between results obtained with both calibrations for all images and reconstruction methods were smaller than $0.5 \%$ for the SUV ratios and $2-5 \%$ for the SUV values.

\section{B. SUVS computation}

Count rate for the acquired rat data varied from 8 to $50 \mathrm{kcps}$ depending on the particular acquisition. Specific rates on the reconstructed images ranged from 20 to 95 $\mathrm{kcps} / \mathrm{cc}$ (3D-OSEM) or 6 to $19 \mathrm{kcps} / \mathrm{cc}$ (improved 3DOSEM) for the different acquisitions and regions of the image. These values are well within the range of the calibration data acquired as we can see in Fig. 3. 
TABLE II

STANDARD UPTAKE VALUES FOR 3D-OSEM METHOD

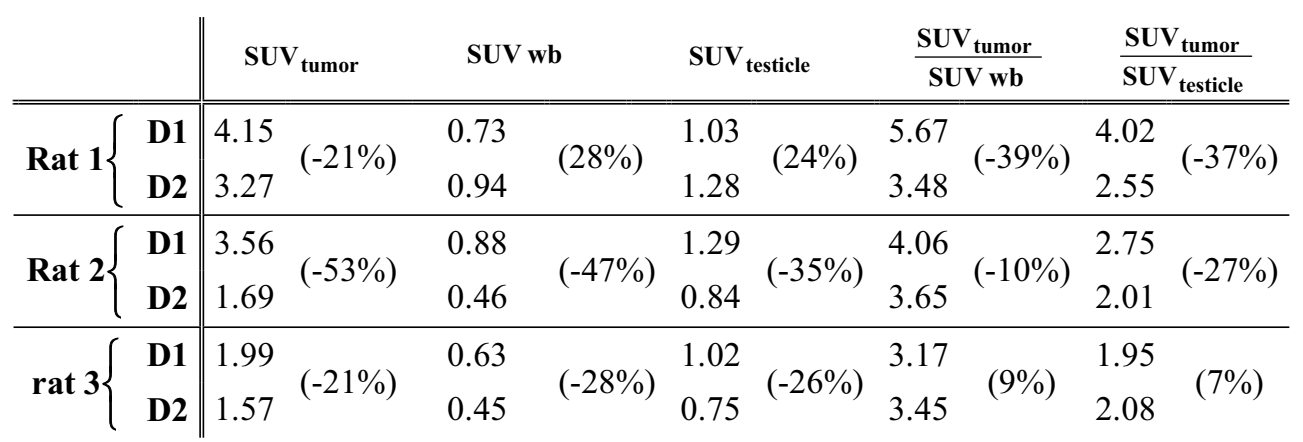

TABLE III

STANDARD UPTAKE VALUES FOR IMPROVED 3D-OSEM METHOD

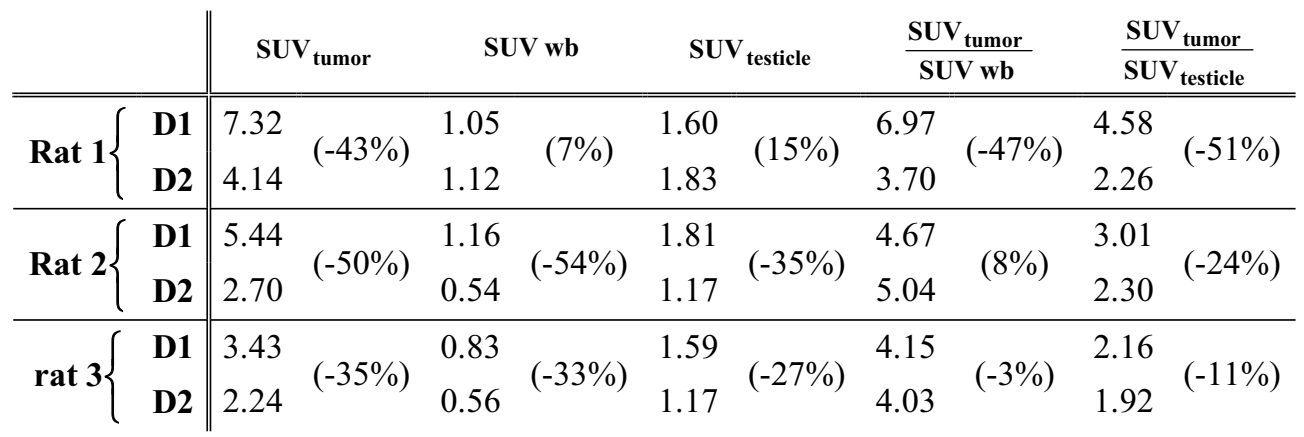

Results for $\mathrm{SUV}_{\text {tumor }}, \mathrm{SUV}_{\mathrm{wb}}$ and $\mathrm{SUV}_{\text {testicle values }}$ and the ratios $\mathrm{SUV}_{\text {tumot }} / \mathrm{SUVwb}$ and $\mathrm{SUV}_{\text {tumor }} / \mathrm{SUV}_{\text {testicle }}$ are presented in tables II and III. Relative variations pre-post therapy of the SUV values (shown inside round parenthesis in the tables) were also computed with the following expression:

$$
\Delta=\frac{\operatorname{SUV}(\mathrm{D} 2)-\operatorname{SUV}(\mathrm{D} 1)}{\operatorname{SUV}(\mathrm{D} 1)} \times 100
$$

The results obtained for the $\mathrm{SUV}_{\text {tumor }} / \mathrm{SUV}_{\text {testicle }}$ ratios are also plotted in Fig. 4.

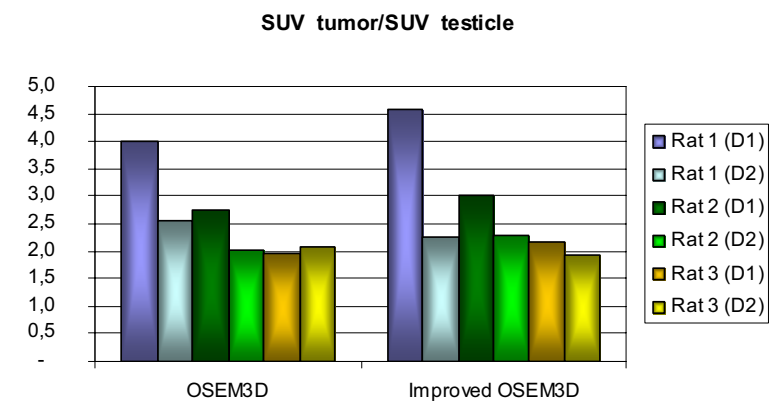

Fig. 4. SUV(tumor) / SUV(testicle) measured ratios on 3D-OSEM and improved 3D-OSEM methods. SUV values of pre-therapy day (D1) and post-therapy day (D2) are shown.

\section{CONCLUSIONS}

From the results presented in Fig. 3 and 4 and tables II and III, it is clearly seen that:

I) On average, both OSEM3D and improved OSEM3D PET images are able to assess positive response of tumor to treatment.

II) Linear and non linear calibrations yield essentially identical results for the improved 3D-OSEM images, with differences smaller than $5 \%$ for the SUV values and than $0.5 \%$ for the SUV ratios. This indicates the ability of the improved reconstruction of reducing non-linear contributions to the scanner response.

III) A linear calibration is suitable for 3D-OSEM even when no random, scatter and pile-up corrections are incorporated provided that the analysis is restricted to moderate values of activity $(<60 \mathrm{muCi} / \mathrm{cc}$ in our case) were quadratic terms are less important. This reduces the deviations of linear and non-linear fits from $11 \%$ (when linear fit is computed from the whole calibration data range) to $2 \%$ (when linear fit is calculated from low activity concentrations) for the SUV values and inferior to $0.5 \%$ for the SUV ratios.

IV) The ratio of $\mathrm{SUV}_{\text {tumor }} / \mathrm{SUV}_{\text {testicle }}$ seems to be appropriate for assessing the response of tumors to treatment.

V) The improved OSEM method might provide enhanced lesion recovery. Changes induced by therapy in 
SUV(tumor)/SUV(testicle) values appear 10\% larger, in the average of the three rats, for improved OSEM with respect to standard OSEM. This is in accordance with previous studies on phantoms that also showed increased contrast and larger recovery coefficients for the improved 3DOSEM reconstruction [13].

\section{ACKNOWLEDGMENTS}

This work has been partially funded by projects TEC2004-07052-C02 of Ministerio de Educación y Ciencia and CD-TEAM, program CENIT, Ministerio de Industria, Spain. Part of the computations of this work were done at the "High capacity cluster for physical techniques" of the Faculty for Physical Sciences of the UCM, funded in part by the UE under the FEDER program and in part by the UCM.

\section{REFERENCES}

[1] Biersack HJ, Bender H, Palmedo H. FDG-PET in monitoring therapy of breast cancer. Eur J Nucl Med Mol Imaging 2004, (Suppl 1):S112-S117

[2] Kostakoglu L, Goldsmith SJ. PET in the assessment of therapy response in patients with carcinoma of the head and neck and of the esophagus. J Nucl Med. 2004;45:56 68.

[3] Kostakoglu L, Goldsmith SJ. 18F-FDG PET evaluation of the response to therapy for lymphoma and for breast, lung, and colorectal carcinoma. J Nucl Med. 2003;44:224 239.

[4] Lale Kostakoglu, MD; and Stanley J. Goldsmith, MD. 18F-FDG PET Evaluation of the Response to Therapy for Lymphoma and for Breast, Lung, and Colorectal Carcinoma. J Nucl Med 2003; 44:224 239

[5] Wolfgang A. Weber, MD. Use of PET for Monitoring Cancer Therapy and for Predicting Outcome. J Nucl Med 2005; 46:983 995

[6] Misquitta A, Herman S, Winton T, Burkes R, Shepherd F, Rappaport D. Accuracy of $C T$ in predicting prognosis in patientswith non-small cell lung carcinoma after induction chemotherapy. Radiology 1994; pp. 193: 257

[7] Parrat E, Pujol JL, Gautier V, Michel FB, Godard P. Chest tumor response during lung cancer chemotherapy. Computed tomography vs fiberoptic bronchoscopy. Chest 1993; 103: 14951501.

[8] Pujol JL, Demoly P, Daures JP, Tarhini H, Godard P, Michel FB. Chest tumor response measurement during lung cancer chemotherapy. Comparison between computed tomography and standard roentgenography. Am Rev Respir Dis 1992; 145: 1149 1154.

[9] Bendriem, B.; Townsend, D.W. The Theory and Practice of 3D PET. Developments in Nuclear Medicine, Vol. 32, 1998. Chapter 4.

[10]J. J. Vaquero, E. Lage, L. Ricon, M. Abella, E. Vicente, and M. Desco. rPET Detector Design and Data Processing. 2005 IEEE Nuclear Science Symposium Conference Record, 2005; pp. 28852889.

[11] Vaquero, J.J. Lage, E. Redondo, S. Abella, M. Pascau, J. Sanchez, J. Vicente, E. Soto-Montenegro, M.L. Desco, M. Co-planar PET/CT for small animal imaging. IEEE Nuclear Science Symposium Conference Record, 2005; pp. 1748-1751.

[12] J. L. Herraiz, S. Espana, J. J. Vaquero, M. Desco, and J. M. Udias, FIRST: Fast Iterative Reconstruction Software for (PET) tomography. Phys. Med. Biol., vol. 51, pp. 4547, 2006. Fan-Beam Collimators," Nuclear Science, IEEE Transactions on, vol. 50, 2003 pp. $398-404$

[13]S. España, J. L. Herraiz, E. Vicente, E. Herranz, J. J. Vaquero, M. Desco, J. M. Udías. Improved image reconstruction in small animal PET using a priori estimation of single-pixel events. Nuclear Science Symposium Conference Record, 2007, abstract \#2056,

[14] Joseph A. Thie. Understanding the Standardized Uptake Value, Its Methods, and Implications for Usage. Journal of Nuclear Medicine, vol.45 pp 1431, 2004.
[15]C.J. Hoekstra, I. Paglianiti, O.S. Hoekstra, E.F. Smit, P.E. Postmus, G.J. Teule and A.A. Lammertsma. Monitoring response to therapy in cancer using [18F]-2-Fluoro-2-deoxy-D-glucose and positron emission tomography: an overview of different analytical methods, Eur. J. Nucl. Med. 27 (2000), pp. 731743

[16]Boellaard R, Krak NC, Hoekstra OS, Lammertsma AA. Effects of noise, image resolution, and ROI definition on the accuracy of standard uptake values: a simulation study. J Nucl Med. 2004; 45:1519-1527.

[17] Beaulieu, S. Kinahan, P. Tseng, J. Dunnwald, L. K. Schubert, E. K.; Pham, P. Lewellen, B. Mankoff, D. A. SUV varies with time after inijection in (18)F-FDG PET of breast cancer: characterization and method to adjust for time differences. J Nucl Med 2003; 44: 1044-50. 